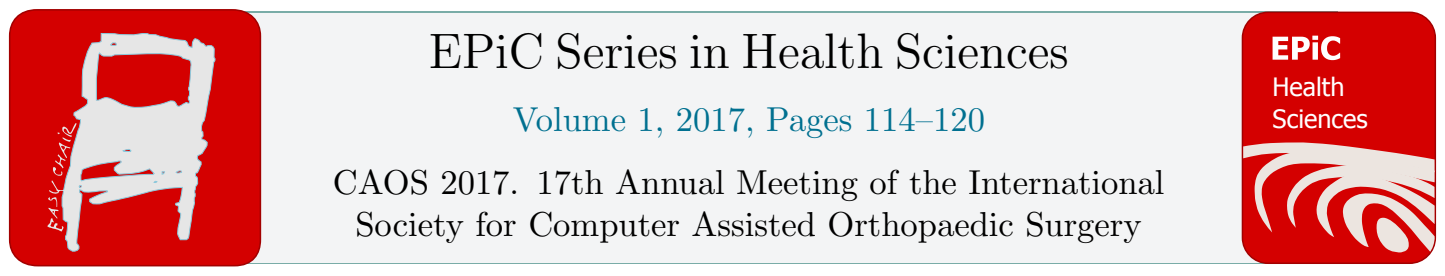

\title{
A New Approach in High Precision Bone Surgery: Semi-Automatic Alignment of an Application-Specific Instrument Guide Adjusted by a Smart Screwdriver
}

\author{
Lukas Theisgen ${ }^{1}$, Sabine Jeromin ${ }^{1}$, Manuel Vossel ${ }^{1}$, Sylvain Billet ${ }^{2}$, Klaus \\ Radermacher ${ }^{1}$ and Matías de la Fuente ${ }^{1}$ \\ ${ }^{1}$ Chair of Medical Engineering, Helmholtz Institute for Biomedical Engineering, RWTH Aachen \\ University, Aachen, Germany \\ ${ }^{2}$ Blue Ortho SAS, La Tronche, France \\ theisgen@hia.rwth-aachen.de
}

\begin{abstract}
Robotic surgical systems reduce the cognitive workload of the surgeon by assisting in guidance and operational tasks. As a result, higher precision and a decreased surgery time are achieved, while human errors are minimised. However, most of robotic systems are expensive, bulky and limited to specific applications.

In this paper a novel semi-automatic robotic system is evaluated that offers the high accuracies of robotic surgery while remaining small, universally applicable and easy to use. The system is composed of a universally applicable handheld device, called Smart Screwdriver (SSD) and an application specific kinematic chain serving as a tool guide. The guide mechanism is equipped with motion screws. By inserting the SSD into a screw head, the screw is identified automatically and the required number of revolutions is executed to achieve the desired pose of the tool guide.

The usability of the system was evaluated according to IEC 60601-1-6 using pedicle screw implementation as an example. The achieved positioning accuracies of the drill sleeve were comparable to those of SpineAssist from Mazor Robotics Ltd., Caesarea (IL) with $-0.54 \pm 0.93 \mathrm{~mm}(\max : 2.08 \mathrm{~mm})$ in medial/lateral-direction and $0.17 \pm 0.51 \mathrm{~mm}(\max : 1.39 \mathrm{~mm}$ ) in cranial/caudal-direction in the pedicle isthmus. Additionally, the system is cost-efficient, safe, easy to integrate in the surgical workflow and universally applicable to applications in which a static position in one or more DOF is to be adjusted.
\end{abstract}




\section{Introduction}

In conventional surgical procedures, the outcome of the operation is influenced by human errors caused by active or passive cognitive mistakes (Bogner 1994). Different computer assisted systems have been introduced in the past, ranging from visual navigation to automatic control by robotic systems, in order to minimise the susceptibility to human errors. By automation the working accuracy has increased (Devito et al. 2010), but also the investment and operative expenditures as well as the system complexity and the footprint (Roser et al. 2013). Furthermore, automatic robotic systems are often more limited to specific surgeries. For the alignment of an implant or a surgical tool according to a pre- or intraoperatively established surgical plan, a simple mechanical surgical guidance seems to be more suitable than bulky robotic systems. As patient specific templates cannot be adjusted intraoperatively, adjustable kinematics could be the solution.

A novel semi-automatic system that combines the advantages of high accuracies, safety, ease of use and cost-efficiency has been presented at CAOS International Conference 2013, Orlando for total knee arthroplasty (TKA) (de la Fuente et al. 2013). The concept is also suitable to other applications and will be evaluated exemplary for pedicle screw placement in this paper (Jeromin 2017). As shown in Figure 1, the system consists of a universal smart handheld device, called Smart Screwdriver (SSD), and an adjustable, application specific tool guide that is firmly and safely mounted on a rigid reference frame fixed to the patient's back.

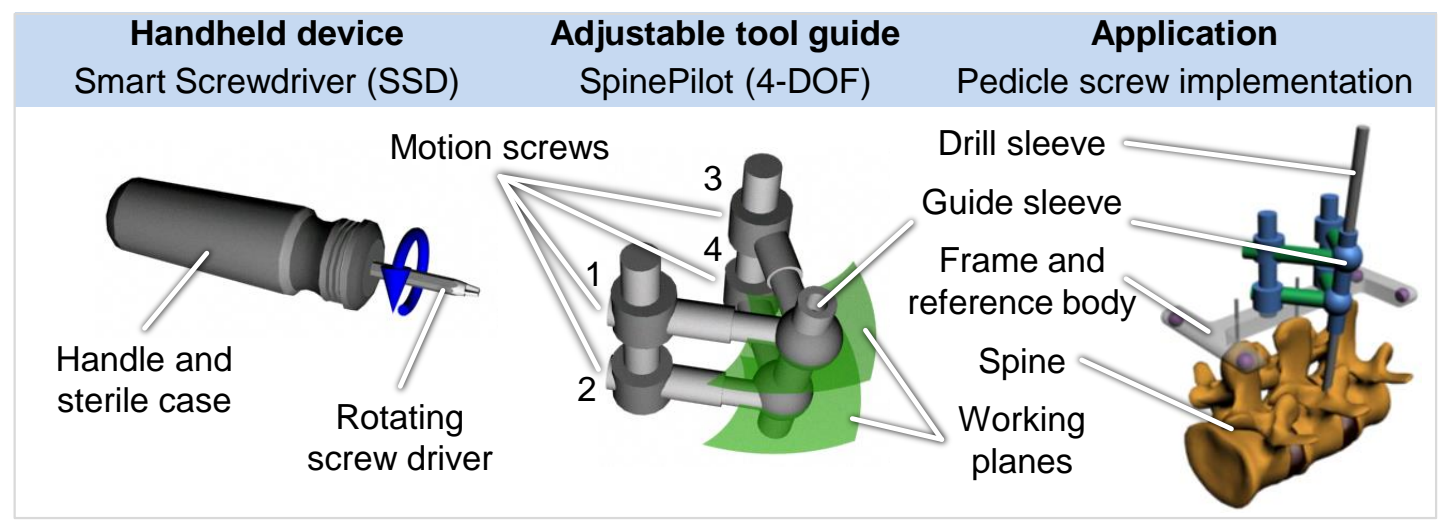

Figure 1: Semi-automatic pedicle screw placement with the SSD and SpinePilot

In case of pedicle screw placement, the tool guide is a four degree of freedom (4-DOF) kinematic chain, called SpinePilot, whereas the SSD is equal for any application. To adjust the position and inclination of the guide sleeve, the SpinePilot is equipped with four motion screws. For automatic identification, each motion screw contains a specific LC circuit. By inserting the SSD into a screw head, the screw is identified and the required number of revolutions, automatically calculated from surgical planning, is executed to reach the desired pose.

\section{Material and Methods}

The usability of the system was evaluated according to the criteria: effectiveness, efficiency, learnability and user satisfaction, as defined in IEC 60601-1-6 and described below. The effectiveness of the SSD was determined on a 1-DOF test bench by measuring the adjustment accuracy of a single motion screw. A precision encoder served to count the number of executed revolutions. The 
effectiveness of the SpinePilot was examined on a 4-DOF test bench with four adjustable motion screws. Here, the accuracy was measured by an optical tracking system. Both trials were performed by 10 novices and an experienced user. Novices are technically skilled persons that are not familiarised with the system. The experienced user is the engineer who developed the system. Trials regarding the efficiency, learnability and user satisfaction were performed by 10 novices directly on the 4 DOF test bench.

The criterion effectiveness was fulfilled if the task could always be completed and if the accuracy in position and inclination was sufficient for transpedicular instrumentation. The pedicle diameter and its surrounding epidural space were used to define the required accuracy. For most vertebrae the epidural space allows a breach of $2 \mathrm{~mm}$ beyond the medial border without complication (Gertzbein and Robbins 1990). In the upper thoracic vertebrae the safety area is $1 \mathrm{~mm}$ (Mirza et al. 2003). According to (Rampersaud et al. 2000) the diameter of a standard pedicle screw is $1 \mathrm{~mm}$ smaller than the pedicle width. The resulting required system accuracy and the achievable accuracy with a robotic guiding system in the pedicle isthmus is $\pm 1.5 \mathrm{~mm}$ (Lieberman et al. 2012).

The efficiency of the design was determined by the time the novices needed for the adjustment procedure. Following (Laine et al. 2000), surgeons need approximately 5 min to implant a pedicle screw under X-Ray control. Assuming that drilling through the sleeve and pedicle screw insertion take $1 \mathrm{~min}$ respectively, 3 min remain for the adjustment procedure.

We measured the learnability by counting the number of repetitions that the novices needed to achieve the highest effectiveness and efficiency. A high learning curve, represented by a success rate of $100 \%$ after a training procedure of not more than five simulated pose adjustments, defines a good learnability.

For the evaluation of the user satisfaction, a questionnaire was established according to ISO 9241/110. The questionnaire comprises 14 statements focussed on the workload and the ergonomic design of the SSD, the SpinePilot and the software (Brooke 1996). Each statement was to be ranked by one to five points. Zero points correspond to a negation of the statement; five points represent a high agreement.

In addition to the evaluation of the usability, the risk analysis method mAIXuse (Janß et al. 2011) and the risk management software CARAD from SurgiTAIX AG, Aachen were applied to evaluate the patient's safety.

\section{Results}

In the first trial with the 1-DOF test bench, the adjustment error of one motion screw, calibrated to the users, was $0 \pm 5.94^{\circ}\left(\max : 15.85^{\circ}\right)$ by the novices and $0 \pm 2.94^{\circ}\left(\max : 8.33^{\circ}\right)$ by the technical expert. Assuming that four motion screws show the same errors, the estimated resulting mean positioning error in the pedicle isthmus was up to $0.58 \mathrm{~mm}$ by the novices and up to $0.3 \mathrm{~mm}$ by the expert.

The resulting positioning errors with the 4-DOF test bench are provided in Figure 2. The novices positioned the pedicle screws with a mean accuracy of $0.54 \pm 0.93 \mathrm{~mm}(\max : 2.08 \mathrm{~mm})$ in medial/lateral-direction and $0.17 \pm 0.51 \mathrm{~mm}$ (max: $1.39 \mathrm{~mm}$ ) in cranial/caudal-direction. 


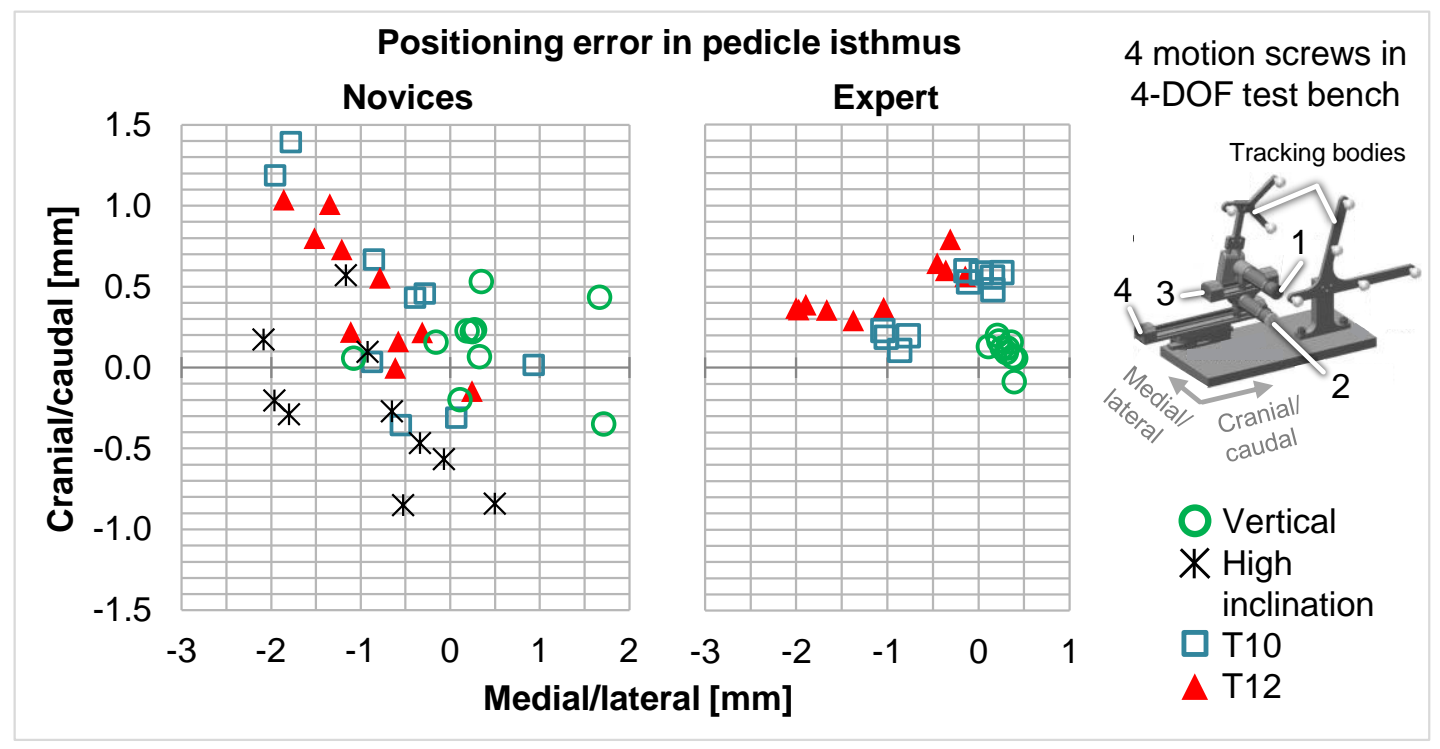

Figure 2: Reached accuracy of the planning transfer

The angular error of the drill axis is not illustrated but was $0.02 \pm 0.27^{\circ}\left(\max : 0.56^{\circ}\right)$ in the transversal and $0.85 \pm 0.30^{\circ}\left(\max : 1.56^{\circ}\right)$ in the sagittal plane by the novices. The expert achieved a mean accuracy of $0.62 \pm 0.31^{\circ}\left(\max : 0.91^{\circ}\right)$ in the transversal and $0.15 \pm 0.08^{\circ}\left(\max : 0.27^{\circ}\right)$ in the sagittal plane.

The interaction time is the time that only the user needs for the adjustment of a pose. The automated screwing is not included. The interaction time decreased with each repetition, as shown in Figure 3. Finally, a novice needed less than $1 \mathrm{~min}$ for a pose.

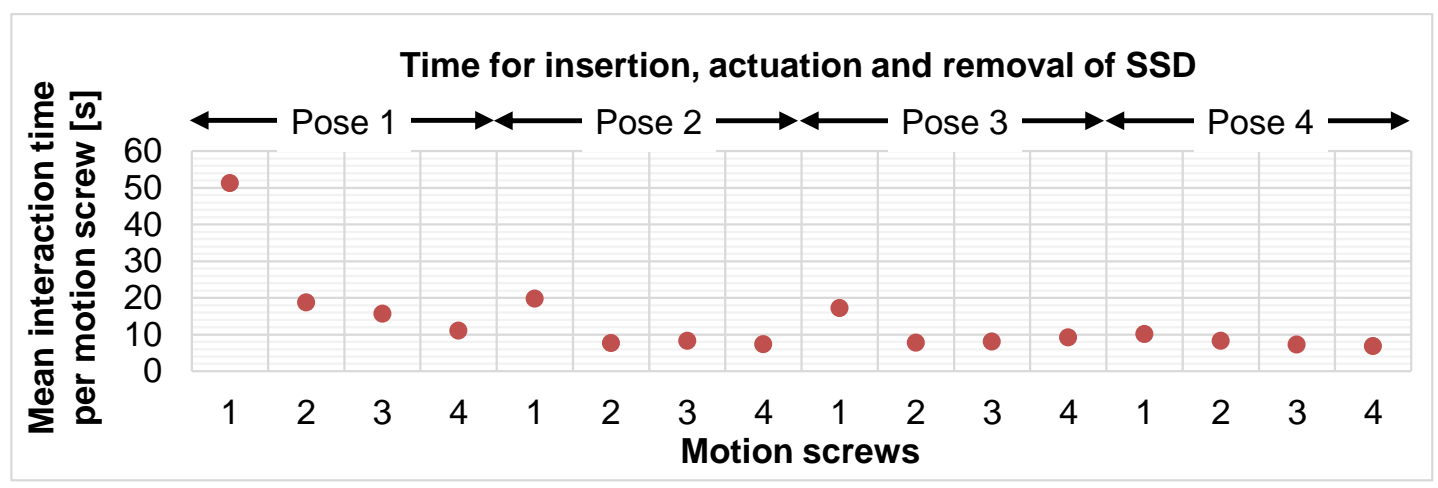

Figure 3: Interaction time for the adjustment of subsequent poses

In Figure 4, the results of the questionnaire are illustrated. The user feedback was overall positive. The risk analysis performed with the mAIXuse method revealed only weak points that can be avoided by modifications in the mechanical design. 


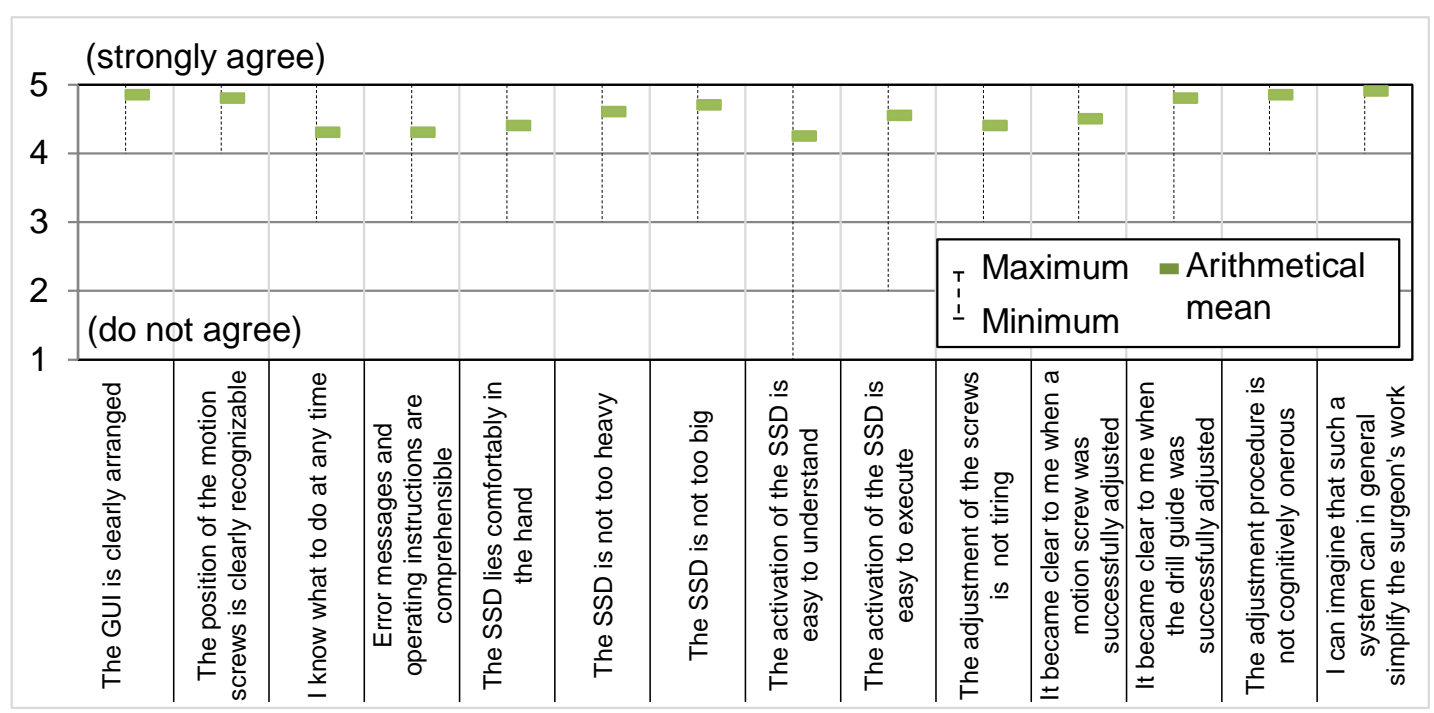

Figure 4: Questionnaire for the user evaluation of the SSD and SpinePilot

\section{Discussion}

The SSD concept represents a novel semi-automatic solution for the planning transfer of various surgical applications. Only passive and purely mechanically moving components are attached to the patient due to the unique structural concept. The system is very safe as the surgeon can interrupt the adjustment procedure by only retracting the SSD from the motion screw. As a result of the modular design, the system is cost-efficient. The technically complex drive module (SSD) is universally applicable and only the specific n-DOF mechanical guide mechanism varies with the application but can be manufactured and reprocessed at low costs.

The presented semi-automatic system has an accuracy in planning transfer that is comparable to that of the miniature semi-automatic guiding robot SpineAssist from Mazor Robotics Ltd., Caesarea (IL), as shown in Table 1. Despite good values for the average positioning error, the maximum value of $2.08 \mathrm{~mm}$ misses the required accuracy of $\pm 1.5 \mathrm{~mm}$. Examinations revealed that inaccuracies in manufacturing may be the reason for such high deviations. Furthermore, only one of 340 poses deviates $2 \mathrm{~mm}$ in the pedicle isthmus whereas in conventional operations an average of $5 \%$ show deviations > $2 \mathrm{~mm}$ (Kosmopoulos and Schizas 2007).

\begin{tabular}{ccc}
\hline Direction & SSD and SpinePilot (Novices) & $\begin{array}{c}\text { SpineAssist from Mazor Robotics } \\
\text { Ltd., Caesarea (IL) }\end{array}$ \\
\hline Medial/lateral & $-0.54 \pm 0.93 \mathrm{~mm}(\max :-2.08 \mathrm{~mm})$ & $0.81 \pm 0.54 \mathrm{~mm}(\max : 1.5 \mathrm{~mm})$ \\
Cranial/caudal & $0.17 \pm 0.51 \mathrm{~mm}(\max : 1.39 \mathrm{~mm})$ & $0.88 \pm 0.65 \mathrm{~mm}(\max : 1.5 \mathrm{~mm})$ \\
\hline
\end{tabular}

Table 1: Comparison of the positioning error between the SSD-SpinePilot system and fully automatic robotic systems according to (Togawa et al. 2007)

Regarding the efficiency, the surgery is faster $(<4$ min per pedicle screw) than with conventional methods (5 min per pedicle screw). By using more than one guide mechanism or more than one SSD simultaneously, the surgery time would be even shorter. 
The learning curve is steep and the efficiency was as high as that of the experienced user after only three repetitions. The handling of the SSD under laboratory conditions was performed intuitively by novices and without any questions to the supervisor. Correspondingly, the user satisfaction was high and suggestions for improvement were limited to simple modifications of the design and the user interface.

As the trials were performed under laboratory conditions, the influence of operating room pollution requires further investigation. Also the structural behavior under mechanical loads, the fixation on the patient and the visual imaging method were not part of the research and are to be examined.

In conclusion, the presented semi-automatic SSD and guiding mechanism is suitable in general for all applications in which a static position in one or more DOF is to be adjusted. Further surgical applications that do not require dynamic instrument guidance should be tested with the novel concept.

\section{References}

- Bogner MS, Human error in medicine, L. Erlbaum Associates, Hillsdale (USA), 1994.

- Brooke J, SUS - A quick and dirty usability scale. In: Jordan PW (ed.): Usability evaluation in industry, Taylor \& Francis, London (GB)/Bristol (USA), pp. 189-194, 1996.

- de la Fuente M, Jeromin S, Boyer A, Billet S, Lavallee S, Stiehl J, Radermacher K, Smart screwdriver for accurate intuitive surgery using the Exactech GPS system, Bone \& joint journal: orthopaedic proceedings supplement, 95(Supp. 28), pp. 83, 2013.

- Devito DP, Kaplan L, Dietl R, Pfeiffer M, Horne D, Silberstein B, Hardenbrook M, Kiriyanthan G, Barzilay Y, Bruskin A, Sackerer D, Alexandrovsky V, Stuer C, Burger R, Maeurer J, Donald GD, Schoenmayr R, Friedlander A, Knoller N, Schmieder K, Pechlivanis I, Kim I-S, Meyer B, Shoham M, Clinical acceptance and accuracy assessment of spinal implants guided with SpineAssist surgical robot: retrospective study, Spine, 35(24), pp. 2109-2115, 2010.

- Gertzbein SD, Robbins SE, Accuracy of pedicular screw placement in vivo, Spine, 15(1), pp. 11-14, 1990.

- Janß A, Lauer W, Pekam FC, Radermacher K, Using new model-based techniques for the user interface design of medical devices and systems, pp. 234-251, 2011.

- Jeromin S, Interaktive planungsgenaue Justierung passiver chirurgischer Instrumentenführungen mittels computergesteuerter Universalstellantriebe, Dissertation, RWTH Aachen University, 2017.

- Kosmopoulos V, Schizas C, Pedicle screw placement accuracy: a meta-analysis, Spine, 32(3), pp. E111-20, 2007.

- Laine T, Lund T, Ylikoski M, Lohikoski J, Schlenzka D, Accuracy of pedicle screw insertion with and without computer assistance: a randomised controlled clinical study in 100 consecutive patients, Eur Spine J, 9(3), pp. 235-240, 2000.

- Lieberman IH, Hardenbrook MA, Wang JC, Guyer RD, Assessment of pedicle screw placement accuracy, procedure time, and radiation exposure using a miniature robotic guidance system, J Spinal Disord Tech, 25(5), pp. 241-248, 2012.

- Mirza SK, Wiggins GC, Kuntz C, 4th., York JE, Bellabarba C, Knonodi MA, Chapman JR, Shaffrey CI, Accuracy of thoracic vertebral body screw placement using standard fluoroscopy, fluoroscopic image guidance, and computed tomographic image guidance: a cadaver study, Spine, 28(4), pp. 402-413, 2003.

- Rampersaud YR, Foley KT, Shen AC, Williams S, Solomito M, Radiation exposure to the spine surgeon during fluoroscopically assisted pedicle screw insertion, Spine, 25(20), pp. 2637-2645, 2000. 
- Roser F, Tatagiba M, Maier G, Spinal robotics: current applications and future perspectives, Neurosurgery, 72 (Suppl. 1), pp. 12-18, 2013.

- Togawa D, Kayanja MM, Reinhardt MK, Shoham M, Balter A, Friedlander A, Knoller N, Benzel EC, Lieberman IH, Bone-mounted miniature robotic guidance for pedicle screw and translaminar facet screw placement: part 2--Evaluation of system accuracy, Neurosurgery, 60(2, Suppl. 1), pp. ONS129-139, 2007.

\section{Disclosures}

This work has been supported partly by Blue Ortho SAS, La Tronche, France. 